\title{
Comment on "A systematic evaluation of YouTube as an information source for male infertility"
}

\author{
Murat Gul $^{1} \cdot$ Mehmet Kaynar ${ }^{1}$
}

Received: 14 June 2020 / Accepted: 9 July 2020 / Published online: 18 July 2020

(c) Springer Nature Limited 2020

As of 2020, YouTube reached a daily of one billion hours of watch. Every minute, $500 \mathrm{~h}$ of video are uploaded to YouTube [1]. These amazing stats, naturally, bring the increasing number of videos pertained to various medical topics online. However, the current evidence revealed that patients are being exposed to poor quality, biased, and/or commercial content, which may lead to hazardous consequences [2-4]. So far, only a few studies evaluated the quality of medical information about male sexual and reproductive health on YouTube videos [5, 6].

Recently, Ku et al. [7] sought to provide insight into the content of YouTube videos on male infertility. The authors identified the top 50 videos using a search term "male infertility" and assessed the videos by a checklist adapted from American Urological Association guidelines referring to male infertility. Then a Grading system ranging from A to D was created based on checklist scores while Grade A represented the highest information density, Grade D corresponded to the lowest information density. Unexpectedly, higher grades were associated with shorter video duration. The most discussed topics were the definition of infertility (93\%) and different diagnostic methods (90\%). On the other hand, surgical intervention methods (33\%) and assisted reproductive technologies $(31 \%)$ were the least mentioned topics. Healthcare organizations produced the highest number of videos with the highest density, yet the variability in the density of videos was also the highest. Astonishingly, compared with Grades A, B, and C, Grade D YouTube videos had the highest number of total view numbers and likes. More striking is perhaps that $90 \%$ of the assessed videos were of low quality.

The current paper shares the inherited limitations of studies evaluating the YouTube healthcare videos, including a lack of unified scoring systems, a lack of defined search criteria, and a

Murat Gul

drmuratgul@hotmail.com

1 Department of Urology, Selcuk University School of Medicine, Konya, Turkey lack of undetermined optimum number of videos to be evaluated. Aside from these drawbacks, Ku et al. provide a global overview of what is returned when people search for "male infertility" on YouTube. Since the male infertility topic consists of many elements, we believe that a topic of more specified search terms could have provided a more informative frame.

Although the list of limitations could be extended here as for every paper, we actually are missing a major point for this paper. The article wakes us up that the world is blossoming into the digital environment. The era where the clinician was the provider of medical information has already long past. The information is now easily accessible and everywhere such as in forums, social platforms, and video streaming services. In this environment, people communicate with each other, share their experiences, and gather around common interests [8]. During the Covid-19 pandemic, we have seen the power of the digital world and how the knowledge disseminated quickly. However, the fact may not be reflected in every aspect thoroughly. As YouTube maintains its leading position as an online streaming service, more people will tend to seek knowledge in videos. This means people may be exposed to both reliable and unreliable information. Whether we want it or not, this transforming process will continue. Therefore, as the medical community, we should embrace this process and be involved to provide more scientific-based and reliable content on different digital platforms.

\section{Compliance with ethical standards}

Conflict of interest The authors declare that they have no conflict of interest.

Publisher's note Springer Nature remains neutral with regard to jurisdictional claims in published maps and institutional affiliations.

\section{References}

1. Press-YouTube. 2020. https://www.youtube.com/about/press/. Accessed 25 May 2020. 
2. Sajadi KP, Goldman HB. Social networks lack useful content for incontinence. Urology. 2011;78:764-7.

3. Loeb S, Sengupta S, Butaney M, Macaluso JN Jr., Czarniecki SW, Robbins R, et al. Dissemination of misinformative and biased information about prostate cancer on YouTube. Eur Urol. 2019;75:564-7.

4. Madathil KC, Rivera-Rodriguez AJ, Greenstein JS, Gramopadhye AK. Healthcare information on YouTube: a systematic review. Health Inform J. 2015;21:173-94.

5. Gul M, Diri MA. YouTube as a source of information about premature ejaculation treatment. J Sex Med. 2019;16: $1734-40$.
6. Kelly-Hedrick M, Grunberg PH, Brochu F, Zelkowitz P. "It's totally okay to be sad, but never lose hope": content analysis of infertility-related videos on YouTube in relation to viewer preferences. J Med Internet Res. 2018;20:e10199.

7. $\mathrm{Ku} \mathrm{S}$, Balasubramanian A, Yu J, Srivatsav A, Gondokusumo J, Tatem JA, et al. A systematic evaluation of YouTube as an information source for male infertility. Int J Impot Res. 2020. https://doi. org/10.1038/s41443-020-0322-9.

8. Gul M, Huynh LM, El-Khatib FM, Yafi FA, Serefoglu EC. A qualitative analysis of Internet forum discussions on hard flaccid syndrome. Int J Impot Res. 2019. https://doi.org/10.1038/s41443019-0151-x. [Epub ahead of print]. 